\title{
The Influence of Fat Suppression Technique on Diffusion-weighted (DW) MRI in Lung Cancer
} \author{
O'Brien ${ }^{2}$; V Morgan ${ }^{1}$; D Collins ${ }^{1}$; NM deSouza ${ }^{1}\left(^{*}=\right.$ joint first authors) \\ ${ }^{1}$ CRUK Cancer Imaging Centre, Institute of Cancer Research, Downs Road, Surrey SM2 5PT, UK. \\ UK. \\ A Weller, alweller@gmail.com \\ MV Papoutsaki, Vasia.Papoutsaki@icr.ac.uk \\ M Orton, Matthew.Orton@icr.ac.uk \\ M Blackledge, Matthew.Blackledge@icr.ac.uk \\ D Ap Dafydd, derfelapdafydd@hotmail.com \\ C Kelly-Morland, Christian.Kelly-Morland@rmh.nhs.uk \\ Mary O’Brien, Mary.O’Brien@rmh.nhs.uk \\ V Morgan, Veronica.Morgan@rmh.nhs.uk \\ D J Collins, David.Collins@icr.ac.uk \\ N M deSouza, Nandita.Desouza@icr.ac.uk
}

A Weller*1; MV Papoutsaki*1; M Orton"; M Blackledge1; D Ap Dafydd ${ }^{2}$; C Kelly-Moreland ${ }^{2}$; Mary

${ }^{2}$ Departments of Radiology and Medicine, Royal Marsden NHS Foundation Trust, Downs Road, Surrey SM2 5PT,

\section{ABSTRACT}

Purpose: To qualitatively and quantitatively investigate the effect of common vendor-related sequence variations in fat suppression techniques on the diagnostic performance of free-breathing DW protocols for lung imaging.

Methods: 8 patients with malignant lung lesions were scanned in free breathing using two diffusion-weighted (DW) protocols with different fat suppression techniques: DWA used short-tau inversion recovery (STIR), and DWB used Spectral Adiabatic Inversion Recovery (SPAIR). Both techniques were obtained at two time points, between 1 hour and 1 week apart. Image quality was assessed using a 5-point scoring system. The number of lesions visible within lung, mediastinum and at thoracic inlet on the DW $\left(b=800 \mathrm{~s} / \mathrm{mm}^{2}\right)$ images was compared. Signal-to-noise ratios (SNR) were calculated for lesions and para-spinal muscle. Repeatability of ADC values of the lesions was estimated for both protocols together and separately.

Results: There was a signal void at the thoracic inlet in all patients with DWB but not with DWA. DWA images were rated significantly better than DWB images overall quality domains. (Cohen's $\mathrm{K}=1$ ). Although 8 more upper mediastinal/thoracic inlet lymph nodes were detected with DWA than DWB, this did not reach statistical significance $(p=$ 0.23). Tumour ADC values were not significantly different between protocols $(p=0.93)$, their ADC reproducibility was satisfactory $(\mathrm{CoV}=7.7 \%)$ and repeatability of each protocol separately was comparable $\left(\mathrm{CoV}_{\mathrm{DWA}}=3.7 \%(95 \% \mathrm{Cl} 2.5-\right.$ $7.1 \%)$ and $\left.\mathrm{CoV}_{\mathrm{DWB}}=4.6 \%(95 \% \mathrm{Cl} 3.1-8.8 \%)\right)$.

Conclusion: In a free-breathing DW-MRI protocol for lung, STIR fat suppression produced images of better diagnostic quality than SPAIR, while maintaining comparable SNR and providing repeatable quantitative ADC acceptable for use in a multicentre trial setting.

\section{Keywords}

Lung Cancer. Apparent Diffusion Coefficient (ADC). Fat suppression technique. STIR. SPAIR. Diffusion-weighted MRI

\section{Academic Discipline And Sub-Disciplines}

Medical Physics; Mediacal imaging; Cancer imaging; Oncology; Magnetic Resonance Imaging

\section{SUBJECT CLASSIFICATION}

Magnestion Resonance Imaging (MRI), image acquisition parameters and image quality.

\section{STUDY TYPE}

Prospective case series study.

\section{INTRODUCTION}

The poor survival of lung cancer means that its effective treatment is one of the greatest unmet clinical needs in modern oncology (1). In order to improve outcomes and increase efficiency of clinical trials of new drugs, biomarker development is essential (2). Of the imaging biomarkers available, the apparent diffusion coefficient (ADC) derived from diffusion weighted (DW) MRI, has been used increasingly in other tumour types for both lesion characterisation and treatment response evaluation $(3,4)$. In lung cancer, it has also has been reported to correlate well with subsequent response on CT 


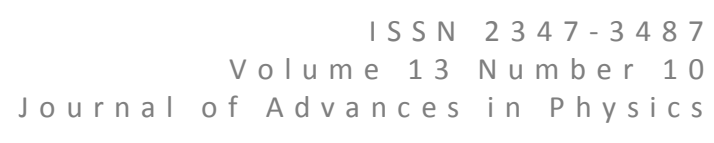

$(5,6)$. However, DW-MRI faces several challenges within the chest, due to low pulmonary parenchymal proton density, respiratory and cardiac motion and magnetic field inhomogeneity, which result in signal voids, ghosting artifact and image distortions (7). Therefore DW-MRI signal to noise ratio (SNR) and diagnostic image quality must be optimised to achieve ADC measurement precision (repeatability and reproducibility) and allow meaningful use in lung imaging. Moreover, in multi-centre clinical trials, there is need for simple, repeatable and standardized protocols. This is not easy to achieve when considering inter-vendor variations that affect diagnostic image quality and ADC measurement precision.

Recently, a robust free breathing protocol has been proposed, showing satisfactory ADC reproducibility, but there are no studies that have investigated the influence of fat suppression technique on measurement precision (8). Therefore, the aim of this study was to investigate qualitatively and quantitatively the effect of common vendor-related sequence variations in fat suppression techniques on the diagnostic performance and ADC quantitation of free-breathing DW protocols for lung imaging, to facilitate the adoption of this biomarker into multi-center clinical trials of lung cancer.

\section{MATERIALS AND METHODS}

\section{Patients}

This prospective single centre study was performed following local institutional review board approval. Between October 2014 and January 2015 informed written consent was obtained from 10 patients ( 5 men, 5 women, age range 66 - 83 years) with at least one lung tumour larger than $2 \mathrm{~cm}$ and minimum of one week since any anti-tumour therapy. They underwent 2 repeated scans within two visits separated by an interval of between 2 hours and 1 week (mean 1.46 days, sd 2.39 days), to assess repeatability of the ADC measurements.

\section{Imaging}

Quality assurance (QA) measurements were performed using an ice-water phantom, scanned to assess accuracy and repeatability of the ADC estimates (9), and a fat -- water cylindrical phantom to estimate the most appropriate field-of-view (10), at which ADC uniformity was maximised along all co-ordinates.

Thoracic MR imaging was performed on a clinical 1.5T MRI scanner (MAGNETOM, Avanto, Siemens, AG, Enlargen Germany). This included full lung coverage, from apices to posterior costo-phrenic sulci, using $2 x$ 12-channel phasedarrays with anterior and posterior elements. Patients were imaged supine with due care as to consistencies of positioning at each visit, to reduce errors from variable patient positioning.

The imaging protocol comprised both anatomical and DW-MRI. For the anatomic measurements, the following pulse sequences were acquired: (a) Three dimensional (3D) T1-weighted (T1-W) gradient-echo sequence in breath hold; (b) 3D T2-weighted (T2-W) 3D turbo spin-echo sequence with variable flip angle (Sampling Perfection with Application optimised Contrasts using different flip angle Evolution, SPACE), with a respiratory-triggered navigator; and (c) Single Slice dynamic T2-W Steady-State Free Precession (SSFP) sequence in free breathing, to provide a representative $6 \mathrm{~mm}$ coronal slice through the largest lesion.

The DW-MRI images were acquired in the axial plane using two single shot echo-planar pulse sequence protocols, referred herein as DWA and DWB, performed in free breathing (Table 1). The DWA protocol has previously been demonstrated to have satisfactory reproducibility within the chest and employed short-tau inversion recovery (STIR) fat suppression while applying an isotropic diffusion gradient in three orthogonal directions (Siemens 'trace' diffusion-gradient mode) (8). DWB employed Spectral Adiabatic Inversion Recovery (SPAIR) fat suppression, with diffusion gradients applied along each of 3 orthogonal directions independently. Different diffusion gradient modes were employed between protocols to enable a motion correction algorithm if needed. Each DW protocol (DWA and DWB) was repeated four times without signal averaging to account for noise from the patient as well as the acquisition. From the fat suppression phantom measurements, coverage equal to or less than $20 \mathrm{~cm}$ in the cranio-caudal ( $\mathrm{z}$ ) direction ( $\leq 40$ slices of $5 \mathrm{~mm}$ ) provided satisfactory ADC uniformity. This resulted in the use of two scanning volumes for whole lung coverage in most patients (Table 1). 


\begin{tabular}{|c|c|c|}
\hline Protocol & DWA & DWB \\
\hline Respiratory control & Free-breathing & Free-breathing \\
\hline Sequence type & Single shot echo planar imaging & Single shot echo planar imaging \\
\hline Orientation & Axial & Axial \\
\hline Phase-encode direction & Anterior-posterior & Anterior-posterior \\
\hline $\begin{array}{c}\text { Field of view [mm (read) } \times \mathrm{mm} \\
\text { (phase)] }\end{array}$ & $380 \times 273$ & $380 \times 273$ \\
\hline Acquired matrix & $128 \times 92$ & $128 \times 92$ \\
\hline Number of Signal Averages & 1 & 1 \\
\hline Number of measurements & 4 & 4 \\
\hline Slice thickness (mm) & 5 & 5 \\
\hline Number of slices & $\begin{array}{c}25-40 \text { with one of two scanning } \\
\text { volumes }\end{array}$ & $\begin{array}{c}25-40 \text { with one of two scanning } \\
\text { volumes }\end{array}$ \\
\hline Parallel imaging reduction factor & 2 & 2 \\
\hline Echo time (ms) & 72 & 83 \\
\hline Repetition time (ms) & 8500 & 9000 \\
\hline Fat suppression & STIR (Tl=180 ms) & SPAIR \\
\hline Diffusion gradient scheme & Single spin echo & Single spin echo \\
\hline Diffusion-encoding scheme & Three scan trace & Orthogonal directions \\
\hline Diffusion weightings (s/mm²) & $100,500,800$ & $100,500,800$ \\
\hline
\end{tabular}

\section{Qualitative analysis}

\section{Image Quality}

A mono-exponential decay model was applied to produce the calculated ADC maps and computed high b-value DW images, using a Levenberg- Marquardt algorithm (ADEPT; Institute of Cancer Research, London, UK). Two experienced radiologists (DD and CKM) qualitatively evaluated the DW images $\left(b=100,800 \mathrm{~s} / \mathrm{mm}^{2}\right)$ and ADC maps of both DWA and DWB protocols, using a 5 point scoring system in the following domains: (Q1) Signal intensity within tumour and pathological lymph nodes compared to background; (Q2) Conspicuity of mediastinal and thoracic inlet structures; (Q3) Uniformity and efficacy of fat suppression technique; and (Q4) Overall image quality. The separate assessment of the $b=100,800 \mathrm{~s} / \mathrm{mm}^{2}$ and ADC maps ensured that a range of contrast perceptions were evaluated. For each patient, the image series (all images from each sequence) were randomised (DWA and DWB) and presented on a DICOM browser, with a standardised window and level setting (Figure 1). The scorers were blinded to the randomisation and rated each image series, assigning a score of $1-5$ to each image series presented for each patient (where $5=$ sufficient signal to delineate anatomic structures within the lung, mediastinum and thoracic inlet and 1=poor anatomic definition) (Figure 1). All image series were scored relative to this highest scoring series for each patient. An average score for each patient for each protocol (DWA and DWB) was obtained in each of the quality domains and for the overall rating. Differences in the qualitative image scores were assessed using the Wilcoxon matched pairs signed rank test. Cohen's Kappa was calculated for agreement between readers in their overall preference for either DWA or DWB, on a per-question basis, using an average score across all patients for each question as a measure of reader preference for each protocol.

\section{Lesion Detection}

Differences in lesion detection rates between the DWA and DWB protocols were evaluated by an experienced radiologist (AW), counting the number of disease deposits visible on $b=800 \mathrm{~s} / \mathrm{mm}^{2} \mathrm{DW}$ images at the following locations: (a) Primary and secondary lesions within lung parenchyma; (b) Lower mediastinal and hilar lymph nodes (Naruke stations 4-11); (c) Upper mediastinal lymph nodes (Naruke stations 1-3). Visualisation of the normal thyroid gland was recorded as a marker for normal and pathological structures at the thoracic inlet. Anatomic localisation was achieved by contemporaneous reference to the T2-W images. The difference in numbers of lesions detected between the DWA and DWB protocols was assessed using the Wilcoxon matched pairs signed rank test.

\section{Quantitative analysis}

Regions of interest were drawn by an experienced radiologist (AW) using a region growing tool on the computed high-b value images $\left(b=800 \mathrm{~s} / \mathrm{mm}^{2}\right)(11)$, encompassing the largest cross section of the lesion across three contiguous slices. $A$ 


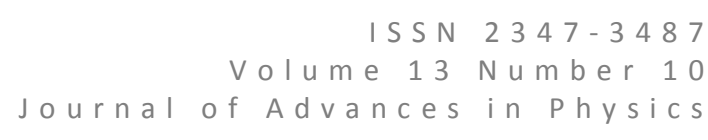

rectangular ROI was drawn on the para-spinal muscle in order to assess the signal to noise ratio (SNR) from a stationary reference tissue. For the three contiguous slices of tumour, the ADC values calculated for all pixels were combined, yielding a volume of interest (VOI) per examination. For each VOI, mean ADC values were recorded and the ADC repeatability of each DW acquisition was evaluated using Bland-Altman plots. The ADC values between the DWA and DWB protocol were compared using Bland-Altman as well as correlation plots.

For both DW protocols, the SNR values within tumour and para-spinal muscle were calculated using the difference method (12). For each ROI, after calculating the mean and difference images of each slice through tumour (or para-spinal muscle) from repeated acquisitions, the mean value of the mean image was divided by the standard deviation of the difference image:

$$
\mathrm{SNR}=(\text { Mean(Meanlmage })) /(\text { StDev(Differencelmage }) / \sqrt{ } 2) \text {. }
$$

The SNR values were reported as decibels using $S N R_{d B}=20 . \log _{10}(S N R)$ as this reduces departures from normality of the SNR distributions, and the distributions were represented graphically with box plots.

\section{RESULTS}

Of the 10 patients recruited and scanned, one was excluded due to the presence of susceptibility artefact at the level of the lesion, more pronounced on DWB than DWA images (as shown in Figure 1) and the other due to failure of the DWA acquisition. Therefore, 8 lesions in 8 patients were included in the analysis; where a patient had multiple lesions (2 patients), the largest was selected.

\section{Qualitative analysis}

\section{Image Quality}

In DWB there was the presence of a signal void at the thoracic inlet, posterior to the manubrium, observed for all patients. This was not present on DWA images (Figure 2). For DWB, this obscured all structures at the thoracic inlet, so that visualisation of the thyroid gland was significantly better with DWA $(p=0.0002)$ (Table 2 and Figure 1).
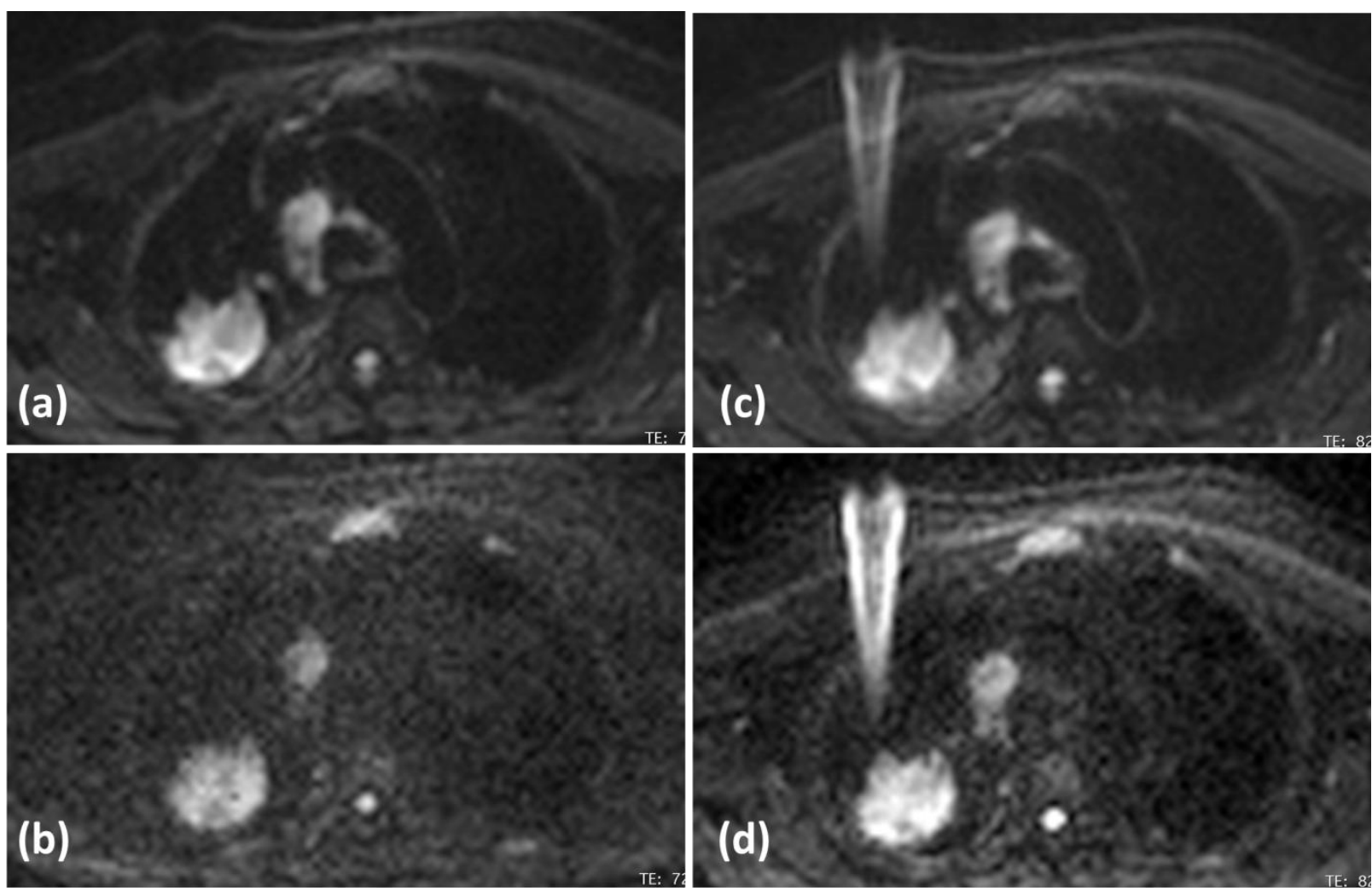

Figure 1: Artefact from a subcutaneous metallic foreign body in the anterior chest wall, causing chemical shift artefact from local magnetic field inhomogeneity, pronounced in the phase encoding direction. Diffusionweighted images of DWA protocol (a) $b=100 \mathrm{~s} / \mathrm{mm}^{2}$ and (b) $b=800 \mathrm{~s} / \mathrm{mm}^{2}$ through the lesion are less vulnerable to image degradation than the corresponding DWB images (c) $b=100 \mathrm{~s} / \mathrm{mm}^{2}$ and (d) $b=800 \mathrm{~s} / \mathrm{mm}^{2}$. As a result of the artefact, this patient was excluded from the analysis. 
Table 2: Lesion detection for different diffusion weighted protocols DWA and DWB, at the four anatomical locations: (a) Primary and secondary lung lesions; (b) Lower mediastinal and hilar lymph nodes (Naruke stations 4-11); (c) Upper mediastinal lymph nodes (Naruke stations 1-3); and (d) Visualisation of the thyroid gland.

\begin{tabular}{|c|c|c|c|}
\hline Lesion detection & $\begin{array}{c}\text { DWA - average } \\
\text { number of lesions } \\
\text { detected per patient }\end{array}$ & $\begin{array}{c}\text { DWB - average } \\
\text { number of lesions } \\
\text { detected per patient }\end{array}$ & $\begin{array}{c}\text { p-value for difference } \\
\text { (Wilcoxon paired signed rank } \\
\text { test) }\end{array}$ \\
\hline $\begin{array}{c}\text { Primary and secondary lung } \\
\text { tumours }\end{array}$ & 2.0 & 2.0 & $\begin{array}{c}\text { Same number of pulmonary } \\
\text { tumours detected }\end{array}$ \\
\hline $\begin{array}{c}\text { Lower mediastinal and hilar } \\
\text { nodes (Naruke 4-11) }\end{array}$ & 1.75 & 1.75 & $\begin{array}{c}\text { Same number of lower med } \\
\text { node detected }\end{array}$ \\
\hline $\begin{array}{c}\text { Upper mediastinal lymph nodes } \\
\text { (Naruke 1-3) }\end{array}$ & 1.88 & 0.75 & 0.23 \\
\hline $\begin{array}{c}\text { Visualisation of thyroid gland } \\
\text { (0=n; } \mathbf{1 = y})\end{array}$ & 0.88 & 0 & 0.0002 \\
\hline
\end{tabular}

Observer 1 rated DWA significantly higher than DWB across domains Q2-Q4 but not in domain 1 (perceived signal intensity within tumour and pathological lymph nodes). Results from observer 2 were largely in agreement, scoring DWA significantly higher than DWB in Q2 (conspicuity of mediastinal and thoracic inlet structures) and Q3 (efficacy of fat suppression), but with no significant difference in scores between DWA and DWB for either Q1 or Q4 (overall image quality). Inter-observer agreement, yielded perfect reader agreement across all questions (Cohen's $\mathrm{k}=1$, Table 3 ). The combined scores (observer 1 plus observer 2) rated DWA as qualitatively significantly better than DWB over all parameters, including for the summated score of all questions. The favourable ranking of DWA over DWB was observed in all domains (Table 3).

Table 3: Individual observer and combined observer scores for image quality across all patients using DW images $\left(b=100,800 \mathrm{~s} / \mathrm{mm}^{2}\right)$ and ADC maps for DWA and DWB protocols. Q1 = Perceived signal intensity within tumour and pathological lymph nodes; Q2 = Conspicuity of mediastinal and thoracic inlet structures; Q3 = Uniformity and efficacy of fat suppression technique; Q4 = Overall image quality.

\begin{tabular}{|c|c|c|c|c|c|c|c|c|c|}
\hline \multirow[t]{3}{*}{ Questions } & \multicolumn{3}{|c|}{ Observer 1} & \multicolumn{3}{|c|}{ Observer 2} & \multicolumn{3}{|c|}{ Observer 1 + Observer 2} \\
\hline & \multicolumn{2}{|c|}{ Protocols } & \multirow[b]{2}{*}{ p-value } & \multicolumn{2}{|c|}{ Protocols } & \multirow[b]{2}{*}{ p-value } & \multicolumn{2}{|c|}{ Protocols } & \multirow[b]{2}{*}{ p-value } \\
\hline & DWA & DWB & & DWA & DWB & & DWA & DWB & \\
\hline Q1 & 4.00 & 3.86 & 0.25 & 4.00 & 3.88 & 0.25 & 4.00 & 3.87 & 0.031 \\
\hline Q2 & 4.00 & 2.75 & $<0.0001$ & 3.00 & 2.50 & 0.0005 & 3.50 & 2.63 & $<0.0001$ \\
\hline Q3 & 4.00 & 2.63 & $<0.0001$ & 4.88 & 3.50 & $<0.0001$ & 4.44 & 3.06 & $<0.0001$ \\
\hline Q4 & 4.00 & 3.00 & $<0.0001$ & 4.00 & 3.88 & 0.25 & 4.00 & 3.44 & $<0.0001$ \\
\hline $\begin{array}{c}\text { Mean Q1- } \\
\text { Q4 }\end{array}$ & 4.00 & 3.06 & $<0.0001$ & 3.97 & 3.44 & $<0.0001$ & 3.98 & 3.25 & $<0.0001$ \\
\hline
\end{tabular}

\section{Lesion Detection}

An effect of the signal void at the thoracic inlet was the higher number of upper mediastinal/thoracic inlet lymph nodes that were detected with DWA (15 lesions in total) compared with DWB (7 lesions in total). However, due to sample size, this difference did not reach statistical significance $(p=0.23)$, likely due to the small average number of nodal tumour deposits present per patient in the cohort $(n=1.36)$. In addition, no significant difference was observed between DWA and DWB for detecting pulmonary tumours and lower mediastinal lymph nodes. 

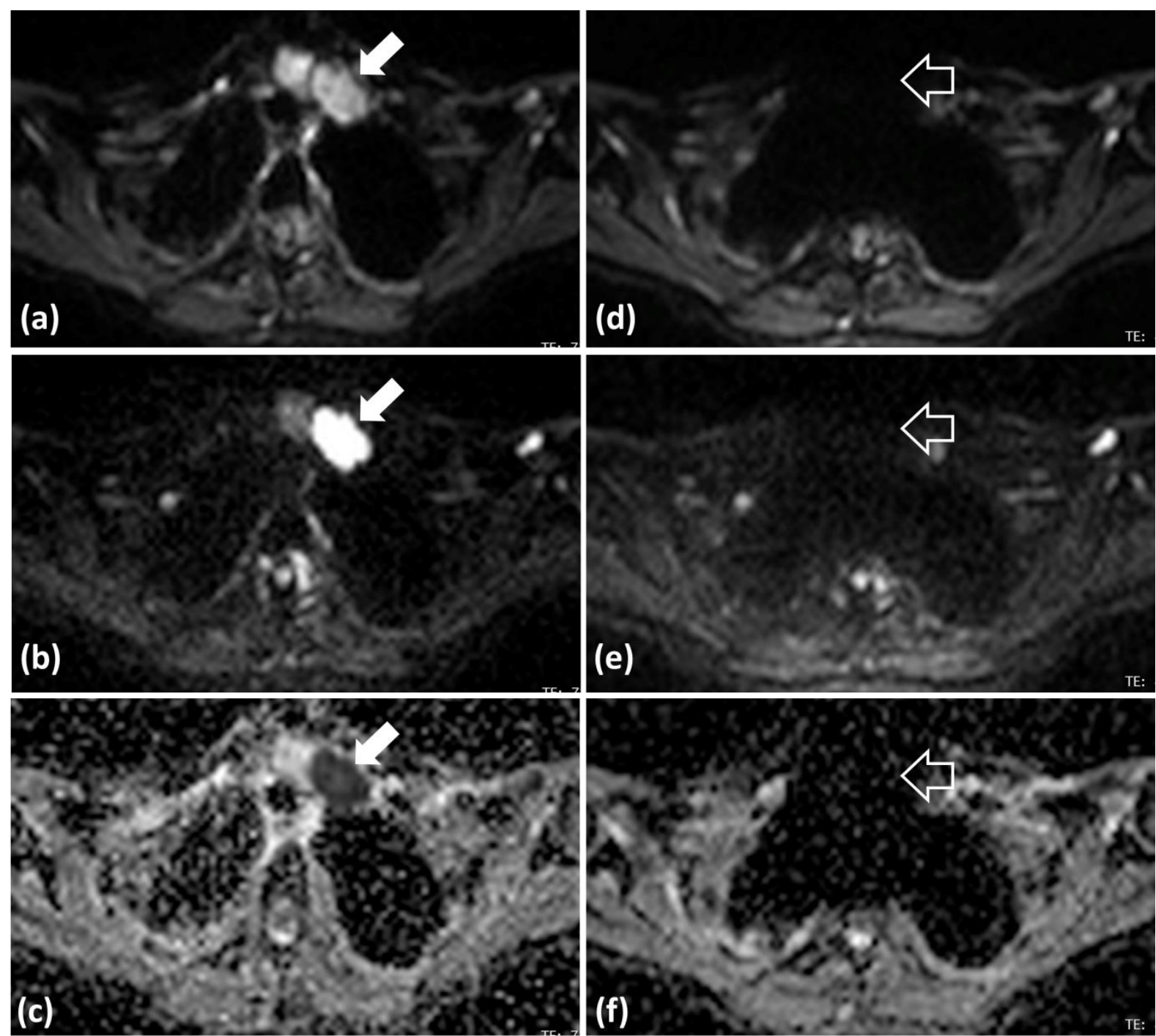

Figure 2: Axial diffusion-weighted $\left(b=100 \mathrm{~s} / \mathrm{mm}^{2}\right.$ and $\left.b=800 \mathrm{~s} / \mathrm{mm}^{2}\right)$ images, with the corresponding apparent diffusion coefficient (ADC) maps through the thoracic inlet on DWA (a-c) and DWB (d-f) protocols. These illustrate the greater conspicuity of supraclavicular nodal disease at the thoracic inlet using DWA protocol (solid arrows). Disease is obscured by signal void when using DWB protocol (hollow arrows).

\section{Quantitative analysis}

Comparison of ADC values for each lesion between DWA and DWB at both scanning visits showed no significant difference in tumour $A D C$ values (t-test $p=0.93$ ) with a mean $A D C$ value $1.14 \pm 0.25 \times 10^{-3} \mathrm{~mm}^{2} / \mathrm{s}$. Pearson's correlation coefficient for ADC between DWA and DWB at both visits was 0.85 (Figure 3). One patient suffered from anxiety at the second scanning visit and gave an outlying result. The centrally located tumour images acquired on this occasion were degraded by respiratory motion resulting in a significantly higher ADC than on the first visit (ADC difference between the two visits $0.345 \times 10^{-3} \mathrm{~mm}^{2} / \mathrm{s}$ ). Reproducibility of the ADC values between the two protocols was reflected by a satisfactory CoV of $7.7 \%$ and removing the outlier improved this to $3.5 \%$. 


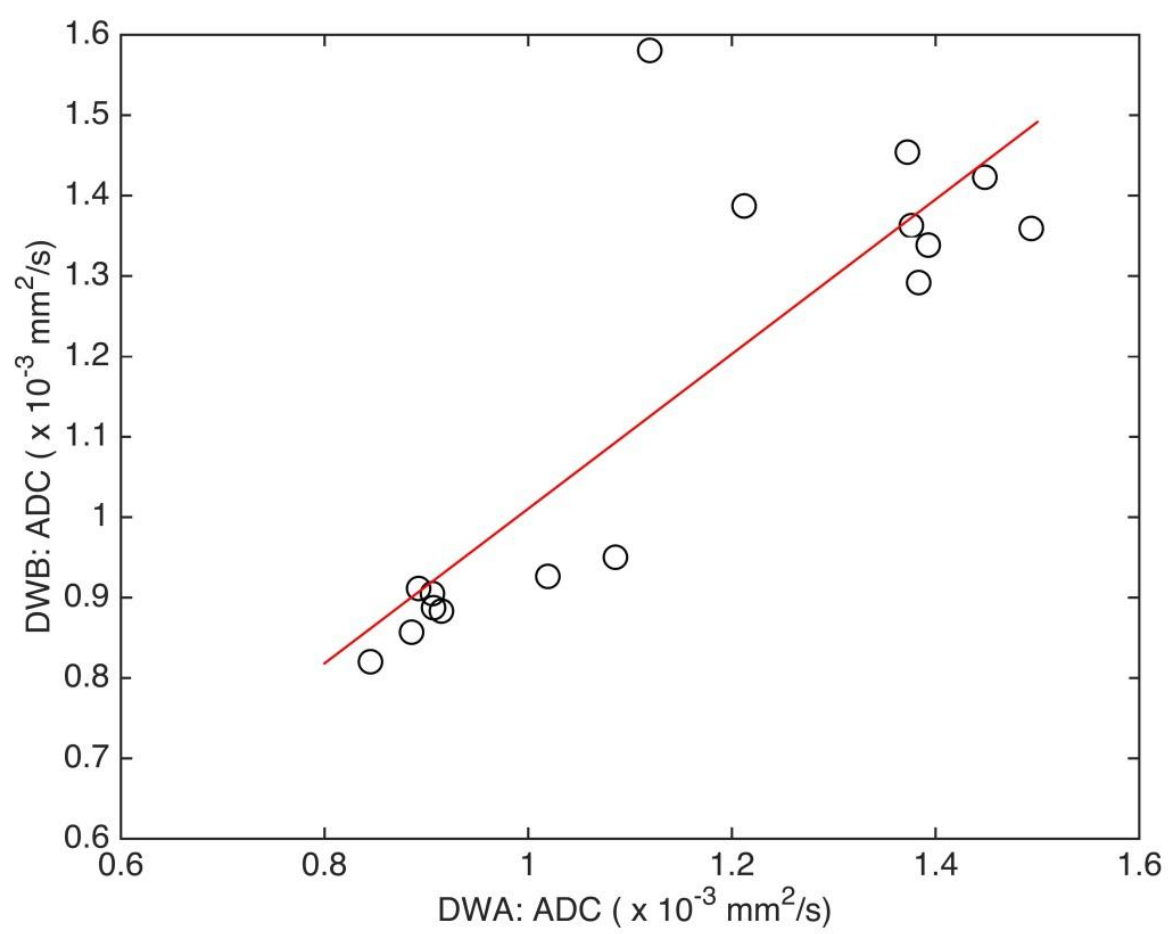

Figure 3: Correlation between the apparent diffusion coefficient (ADC) values from DWA and DWB ( $r=0.85$ ).

Considering repeatability for each of the two protocols, there was no significant difference in mean tumour ADC obtained between the two scanning visits (DWA $p=0.52$, DWB $p=0.58$ ). ADC repeatability for both protocols were comparable; CoVs were $3.7 \%$ for DWA (95\% Cl $2.5-7.1 \%)$ and $4.6 \%(95 \% \mathrm{Cl} 3.1-8.8 \%)$ for DWB and are illustrated by the Bland Altman plots in Figure 4.

(a)

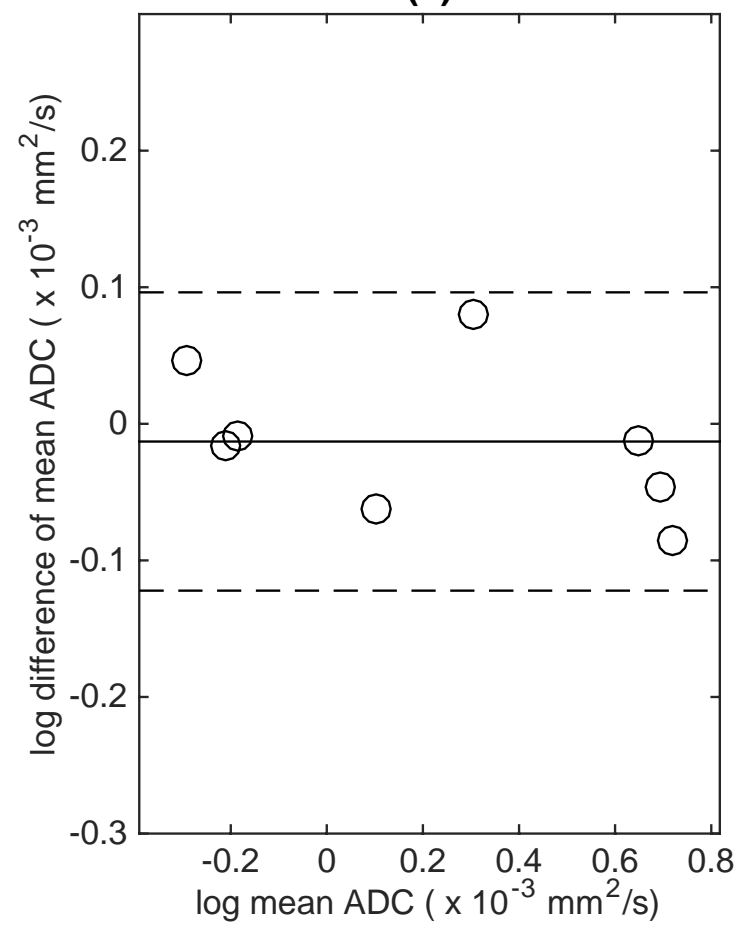

(b)

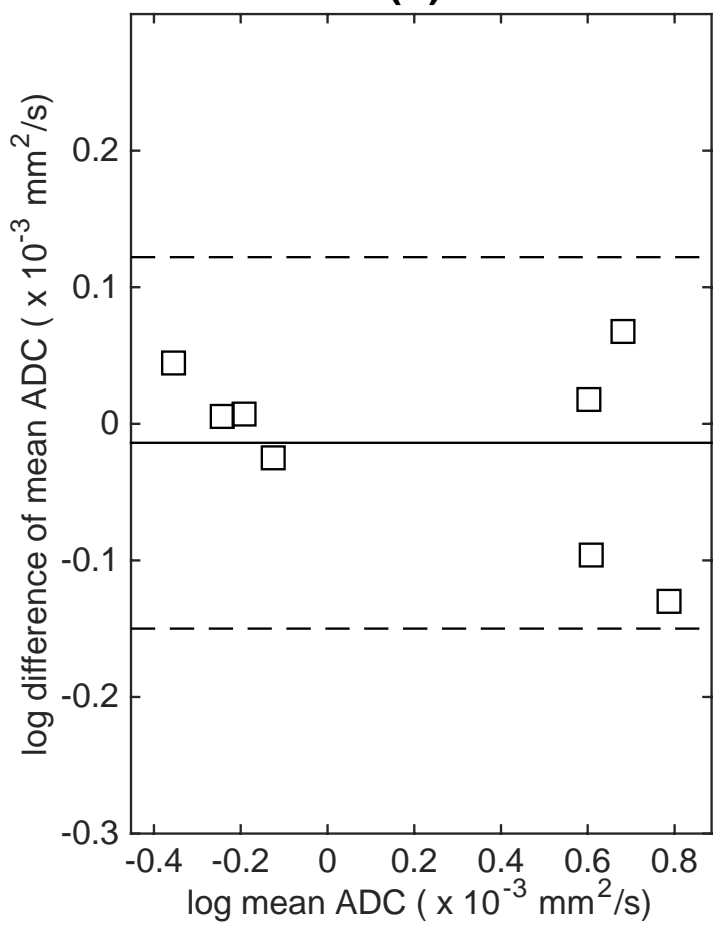

Figure 4: Bland Altman plots showing the repeatability of the apparent diffusion coefficient (ADC) values of DWA (a) and DWB (b). and DWB (b). Dashed lines $95 \%$ limits of agreement. 
SNR estimates derived from tumour and the para-spinal muscle VOls across the DW images $\left(b=100,500,800 \mathrm{~s} / \mathrm{mm}^{2}\right)$ of the two DW protocols are illustrated in Figure 5. For both imaging protocols, SNR decreased with increasing $b$ value as expected. There was no significant difference in SNR from tumour VOls for DWA compared with DWB $(p=0.51)$. However, within muscle a significantly higher SNR was observed for DWB compared with DWA $(p<0.05)$, for all $b$-values (Figure 5$)$. The difference in SNR results between the two anatomical areas of similar T1's reflect the fact that, unlike tumour, muscle is interspersed with fat and therefore likely to be affected by different fat suppression techniques.

(a)

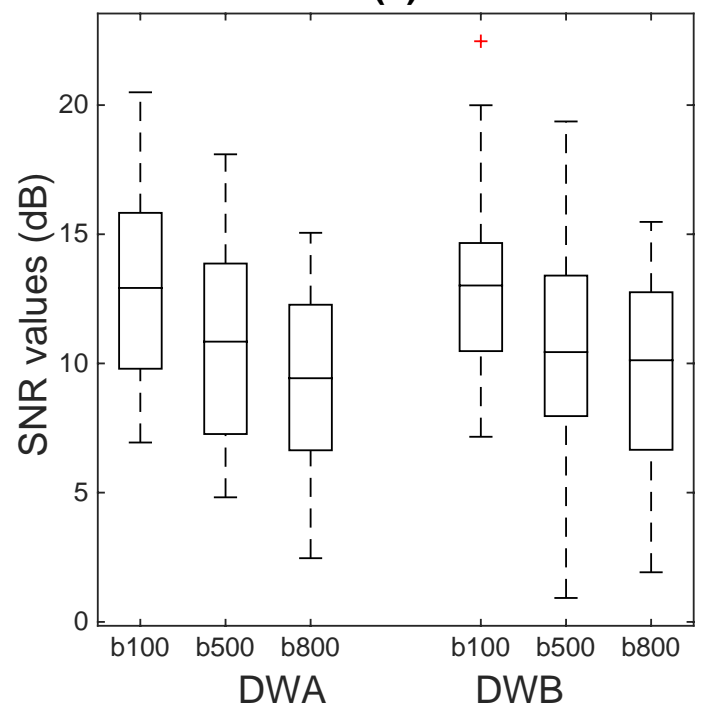

(b)

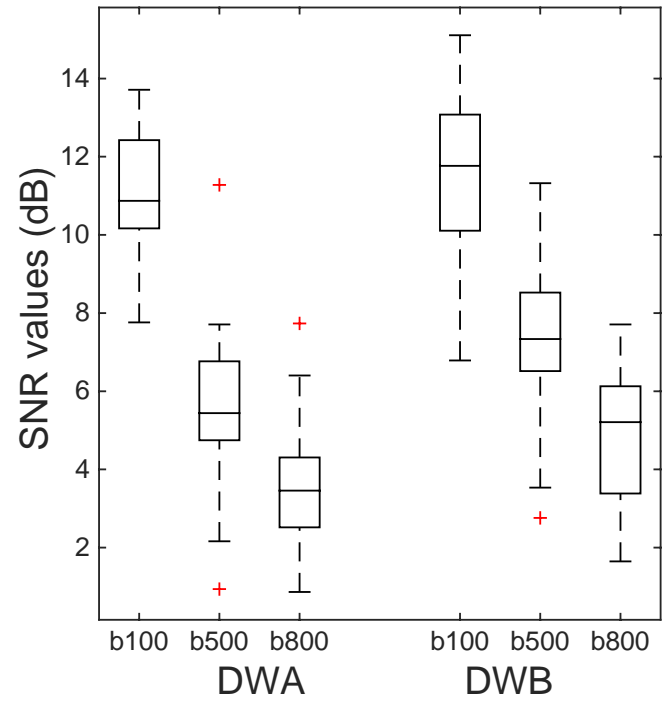

Figure 5: Box plots of the signal to noise ratio (SNR) values from tumour regions of interest (ROI) in tumour (a) and muscle (b) across all the b-values (in $\mathrm{s} / \mathrm{mm}^{2}$ ).

\section{DISCUSSION AND CONCLUSION}

This study demonstrates that an inversion recovery method of fat suppression produces DW-MRI images of superior diagnostic performance compared to spectrally selective methods. Images acquired with STIR fat suppression produced images that had a significantly higher qualitative radiologist preference and enabled superior delineation of anatomical structures at the thoracic inlet. This was achieved without any compromise to ADC quantitation or difference in ADC repeatability. SPAIR fat suppression, on the other hand, resulted in a signal void at the thoracic inlet that obscured the anatomy here. Therefore, when incorporating DW-MRI as a biomarker for monitoring treatment response in multi-centre clinical trials in lung cancer, differences in available fat suppression techniques between vendors need to be considered, as they are likely to influence the reliability of the results.

Effectiveness of the fat suppression is a function of magnetic field homogeneity, which is more critical in spectrally selective methods. Both phantom and volunteer measurements have demonstrated image degradation and loss of effective fat suppression at the edges of the field of view using SPAIR, due to its sensitivity to $B_{0}$ inhomogeneity (10). The use of large fields-of-view to ensure coverage of the thorax meant that the thoracic inlet was at the edge of the field in our patients and resulted in severe signal drop-out. Regions of anatomic transition with sharp boundaries between tissues of different magnetic susceptibilities result in magnetic field inhomogeneity and lead to both signal loss and poorer performance of the SPAIR fat-suppression technique (15). In our cohort, normal thyroid was not visualised for any patients using DWB protocol. This presents a clear limitation when imaging NSCLC, where the presence or absence of supraclavicular nodal disease has a significant impact on patient management - lack of diagnostic confidence in detecting pathology in this location seriously hampers the clinical utility of SPAIR fat suppression (16-18). There is currently no consensus regarding the optimal fat suppression technique for DW-MRI in the chest but our results indicate a radiologist preference for STIR methodology, especially in the upper mediastinum and thoracic inlet.

The robustness of STIR fat suppression, employed in the DWA protocol, has previously been reported, in terms of intraand inter-observer reproducibility (8). Nevertheless, STIR results in lower signal intensity across the field of view due to the inversion pulse, while SPAIR fat-suppression gives higher signal intensity (chemically-selective inversion pulse). The use of different diffusion gradient modes for DWA and DWB meant that we were comparing STIR and SPAIR images with equivalent SNR. For DWB, the application of independent diffusion gradients along 3 orthogonal directions resulted in higher TE and lower signal intensity. Despite this, DWB (SPAIR fat suppression) provided higher SNR within muscle than DWA (STIR) but not within tumour. In addition, no difference was observed qualitatively in perceived signal intensity between the two protocols by scoring radiologists.

The choice of fat suppression technique results in different T1W and T2W contrast contributions to signal (13). STIR introduces a contribution from T1-W contrast while SPAIR contributes both T2-W and T1-W tissue contrast (14). This is not so critical in lung imaging where the tumour is largely surrounded by signal void from air in the lungs. Even in the mediastinum, there is sufficient contrast between a tumour mass and the signal void from flowing blood in the great vessels so that the effect of $\mathrm{T} 1$ and $\mathrm{T} 2-\mathrm{W}$ contrast on the choice of fat suppression is not an issue. The longer acquisition times with STIR fat-suppression also are a disadvantage. 


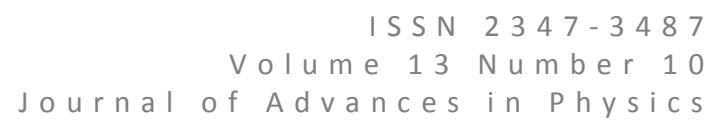

In designing an easily implemented, generalizable and robust DW-MRI protocol, three b-values are recommended for ADC calculation, with a low b (>=100), an intermediate b $\left(400-500 \mathrm{~s} / \mathrm{mm}^{2}\right)$ and a higher b value $\left(500-1000 \mathrm{~s} / \mathrm{mm}^{2}\right)(19)$ We achieved this in an acceptable timeframe for image acquisition despite having to contend with low SNR, magnetic susceptibility and motion effects. Our choice of $b$-values $\left(b=100,500,800 \mathrm{~s} / \mathrm{mm}^{2}\right)$ produced images that were of satisfactory quality and ADC repeatability and had sufficient SNR within both moving (lung lesion) and stationary (paraspinal muscle) structures. The similarity of the SNR calculations from the two protocols (DWA and DWB) and the visualisation of both tumour and abnormal structures on all image series indicate that the $b$-values chosen represent a suitable trade-off between increasing tissue contrast and decreasing signal with increasing b-value.

Respiratory motion can degrade DW-MR images within the chest and this has a variable effect on ADC quantitation (8). As any viable multi-centre trial protocol must be implemented consistently, the most appropriate method for respiratory control within the chest must be considered (19). Techniques other than free breathing lead to variations between institutions, due to the inter-vendor differences in respiratory compensation equipment and acquisition protocols. Respiratory triggering can mitigate motion and preserve signal, but is dependent on a regular respiratory cycle, leading to variability between patients and visits, as well as to long acquisition times $(8,20)$. In addition, this technique has previously shown no advantage over a free breathing protocol for ADC or IVIM quantitation (21). Free breathing protocols with sufficient averaging are versatile and robust for ADC quantification across vendors and institutions $(8,22)$, an observation that is in accordance with the satisfactory ADC repeatability and SNR values presented in our study.

The main limitation of this study was the small number of patients. From the 10 recruited, two were excluded from analysis, each due to severely degraded image quality of one or both of the DW protocols. A second limitation was the fact that DWA and DWB protocols differed not only in fat-suppression technique but also in diffusion gradient mode, TR and $T E$, leading to confounding effects when attempting to compare the performance of different fat-saturation techniques alone. TR was considered sufficiently long to avoid T1w losses in both protocols, the STIR fat suppression will have reduced overall signal in protocol DWA. The different implementation of the diffusion encoding increased the TE in protocol DWB, which would reduce the overall signal through T2 relaxation. In terms of SNR protocol DWB would be improved by using the diffusion encoding and echo time employed in DWA.

In conclusion, our results highlight the importance of a robust and consistent fat-suppression technique when implementing DW-MRI for lung imaging. STIR fat suppression produces images of greater diagnostic quality, delineating structures at the thoracic inlet that are obscured by a signal void when using a spectrally selective (SPAIR) technique. The reproducibility of the quantified ADC however, appears acceptable for use in a multicentre trial setting with both STIR and SPAIR methods of fat suppression.

\section{REFERENCES}

1. Harders SW, Balyasnikowa S, Fischer BM. Functional imaging in lung cancer. Clinical physiology and functional imaging. 2014;34(5):340-55.

2. FDA. Critical Path Opportunities List. http://wwwfdagov/downloads/ScienceResearch/SpecialTopics/CriticalPathInitiative/CriticalPathOpportunitiesReports/UCM 077258pdf. 2006.

3. Bains LJ, Zweifel M, Thoeny HC. Therapy response with diffusion MRI: an update. Cancer imaging : the official publication of the International Cancer Imaging Society. 2012;12:395-402.

4. Charles-Edwards EM. Diffusion-weighted magnetic resonance imaging and its application to cancer. Cancer imaging : the official publication of the International Cancer Imaging Society. 2006;6(1):135.

5. Reischauer C, Froehlich JM, Pless M, Binkert CA, Koh DM, Gutzeit A. Early treatment response in non-small cell lung cancer patients using diffusion-weighted imaging and functional diffusion maps--a feasibility study. PloS one. 2014;9(10):e108052.

6. Yabuuchi H, Hatakenaka M, Takayama K, Matsuo Y, Sunami S, Kamitani T, et al. Non-small cell lung cancer: detection of early response to chemotherapy by using contrast-enhanced dynamic and diffusion-weighted MR imaging. Radiology. 2011;261(2):598-604.

7. Henzler T, Schmid-Bindert G, Schoenberg SO, Fink C. Diffusion and perfusion MRI of the lung and mediastinum. European journal of radiology. 2010;76(3):329-36.

8. Bernardin L, Douglas NH, Collins DJ, Giles SL, O'Flynn EA, Orton M, et al. Diffusion-weighted magnetic resonance imaging for assessment of lung lesions: repeatability of the apparent diffusion coefficient measurement European radiology. 2014;24(2):502-11.

9. Douglas N WJ, deSouza NM, Collins DJ, Orton MO Development of a phantom for quality assurance in multicentre clinical trials with diffusion-weighted MRI. Proceedings of the International Society of Magnetic Resonance in Medicine 2013:Presentation number 3114.

10. Winfield J, Douglas N, Collins D. Phantom for assessment of fat suppression in large field-of-view diffusionweighted magnetic resonance imaging. Physics in medicine and biology. 2014;59(9):2235.

11. Blackledge MD, Leach MO, Collins DJ, Koh DM. Computed diffusion-weighted MR imaging may improve tumor detection. Radiology. 2011;261(2):573-81. 
12. Dietrich O, Raya JG, Reeder SB, Reiser MF, Schoenberg SO. Measurement of signal-to-noise ratios in MR images: Influence of multichannel coils, parallel imaging, and reconstruction filters. Journal of Magnetic Resonance Imaging. 2007;26(2):375-85.

13. Lauenstein TC, Sharma P, Hughes T, Heberlein K, Tudorascu D, Martin DR. Evaluation of optimized inversion-recovery fat-suppression techniques for T2-weighted abdominal MR imaging. Journal of Magnetic Resonance Imaging. 2008;27(6):1448-54.

14. Collins DJ, Blackledge M. Techniques and optimization. Diffusion-Weighted MR Imaging: Springer; 2010. p. 19-

32.

15. Koh D-M, Thoeny HC. Diffusion-weighted MR imaging: applications in the body: Springer Science \& Business Media; 2010

16. Eberhardt WE, De Ruysscher D, Weder W, Le Pechoux C, De Leyn P, Hoffmann H, et al. 2nd ESMO Consensus Conference in Lung Cancer: locally advanced stage III non-small-cell lung cancer. Annals of oncology : official journal of the European Society for Medical Oncology / ESMO. 2015;26(8):1573-88.

17. Reck M, Popat S, Reinmuth N, De Ruysscher D, Kerr KM, Peters S. Metastatic non-small-cell lung cancer (NSCLC): ESMO Clinical Practice Guidelines for diagnosis, treatment and follow-up. Annals of oncology : official journal of the European Society for Medical Oncology / ESMO. 2014;25 Suppl 3:iii27-39.

18. Vansteenkiste J, Crino L, Dooms C, Douillard JY, Faivre-Finn C, Lim E, et al. 2nd ESMO Consensus Conference on Lung Cancer: early-stage non-small-cell lung cancer consensus on diagnosis, treatment and follow-up. Annals of oncology : official journal of the European Society for Medical Oncology / ESMO. 2014;25(8):1462-74.

19. Taouli B, Beer AJ, Chenevert T, Collins D, Lehman C, Matos C, et al. Diffusion-weighted imaging outside the brain: Consensus statement from an ISMRM-sponsored workshop. Journal of magnetic resonance imaging : JMRI. 2016.

20. Deng Y, Li X, Lei Y, Liang C, Liu Z. Use of diffusion-weighted magnetic resonance imaging to distinguish between lung cancer and focal inflammatory lesions: a comparison of intravoxel incoherent motion derived parameters and apparent diffusion coefficient. Acta radiologica (Stockholm, Sweden : 1987). 2015.

21. Jerome NP, Orton MR, d'Arcy JA, Collins DJ, Koh DM, Leach MO. Comparison of free-breathing with navigator-controlled acquisition regimes in abdominal diffusion-weighted magnetic resonance images: Effect on ADC and IVIM statistics. Journal of Magnetic Resonance Imaging. 2014;39(1):235-40.

22. Jerome NP, Orton MR, d'Arcy JA, Feiweier T, Tunariu N, Koh DM, et al. Use of the temporal median and trimmed mean mitigates effects of respiratory motion in multiple-acquisition abdominal diffusion imaging. Physics in medicine and biology. 2015;60(2):N9-N20.

\section{ACKNOWLEDGEMENTS}

We acknowledge CRUK and EPSRC support to the Cancer Imaging Centre at ICR and RMH in association with MRC \& Dept of Health C1060/A10334, C1060/A16464 and NHS funding to the NIHR Biomedical Research Centre and the Clinical Research Facility in Imaging. AW and M-VP were funded by Innovative Medicines Initiative Joint Undertaking under grant agreement number 115151 .

\section{Author biography and photo}

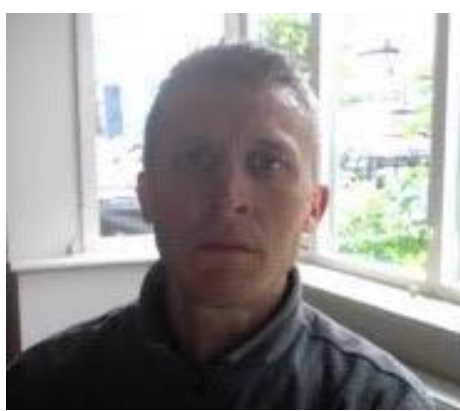

Dr Alexander Weller (FRCR MBBS MChem) is a consultant radiologist at Northwest London NHS Trust, UK and former clinical research fellow in medical imaging at the Institute of Cancer Research (ICR) and Royal Marsden NHS Trust, with interest in functional imaging biomarkers in oncology. His research portfolio includes multicentre evaluation of combined FLT PET/CT and MRI for demonstrating lung cancer treatment response and builds upon a focus on magnentic resonance imaging during specialist training in clinical radiology. This took place at St George's Hospital NHS Trust, with additional experience at Memorial Sloan Kettering Cancer Center, New York. A background masters in neutron scattering (Physical and Theoretical Chemistry Labs, Oxford) prior to his medical education have provided the scientific rigour needed to advance research at the ICR.

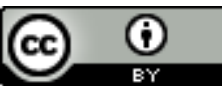

This work is licensed under a Creative Commons Attribution 4.0 International License. 